\title{
O local ideal para a prestação de serviços em alargamento de horário nos cuidados de saúde primários: análise dos custos e da perceção da qualidade dos serviços
}

João Firmino-Machado, ${ }^{1}$ John Yaphe, ${ }^{2}$ Maria José Ribas, ${ }^{3}$ Patrício Costa

\section{RESUMO}

Objetivo: Comparar os custos e as perceções da qualidade dos serviços (PQS) entre os serviços prestados pelas USF e os Serviços de Atendimento de Situações Urgentes (SASU), na região do Porto Ocidental, em alargamento de horário.

Tipo de estudo: Transversal observacional e analítico.

Local: Unidade de Saúde Familiar (USF) Serpa Pinto, USF Ramalde e Serviço de Atendimento de Situações Urgentes (SASU) do Porto, do Agrupamento de Centros de Saúde do Porto Ocidental.

População: Utentes utilizadores do alargamento de horário, nas unidades de saúde USF Serpa Pinto, USF Ramalde e SASU do Porto.

Métodos: A quantificação da PQS foi feita utilizando um instrumento construído para o efeito (SERVQUAL/SERVPERF e suas adaptações à área da saúde - HEALTHQUAL). O questionário foi aplicado a um total de 281 utentes de ambas as unidades que recorreram às unidades de saúde em estudo. A obtenção dos custos de funcionamento (fármacos e meios complementares de diagnóstico) das unidades em alargamento de horário foi feita de forma transversal, por consulta dos processos clínicos de 820 utentes.

Resultados: Os valores globais de PQS nas USF são superiores aos determinados para os SASU, sendo esta diferença estatisticamente significativa, mesmo quando é controlado o peso das características sociodemográficas. Não foram encontradas diferenças estatisticamente significativas entre os custos globais das USF $(€ 9,30 /$ utente, DP=14,9) e do SASU (€ 8,20/utente, $\mathrm{DP}=8,2)$.

Discussão/Conclusão: As USF prestam serviços de saúde com valores de perceção de qualidade de serviços significativamente superiores aos reportados pelos SASU, em regime de alargamento de horário e a custos iguais. Não existe evidência a favor do encerramento dos serviços de alargamento de horário.

Palavras-chave: Satisfação dos doentes; Cuidados de saúde primários; Custos em saúde; Serviço de alargamento de horário.

\section{INTRODUÇÃO}

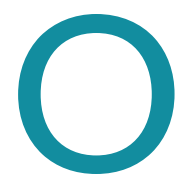

s cuidados de saúde prestados a doentes com doença aguda não urgente representam uma parte significativa dos cuidados de saúde primários, havendo, no entanto, dúvidas sobre qual a forma mais custo-efetiva de prestar este tipo de serviços. ${ }^{1-2}$ Vários modelos têm sido apresentados para o atendimento deste tipo de doentes em regime de alargamento de horário (funciona-

\footnotetext{
1. Unidade de Saúde Pública, ACeS Porto Ocidental

2. Instituto de Investigação em Ciências da Vida e da Saúde (ICVS), Escola de Ciências da Saúde da Universidade do Minho (Braga). ICVS-3Bs, Laboratório Associado do Governo de Portugal, Braga, Guimarães, Portugal

3. Médica de Família. USF Garcia de Orta
} 
mento após as 20h aos dias de semana e aos sábados, domingos e feriados): por médicos de família, nas suas próprias unidades - Unidades de Saúde Familiar (USF) ou noutras unidades de saúde - Serviço de Atendimento de Situações Urgentes (SASU), mas também nos serviços de urgência dos hospitais. ${ }^{1-5}$ Recentes alterações nas políticas de saúde na região do Porto Ocidental proporcionaram uma oportunidade de levar a cabo estudos sobre esta questão. $O$ termo do horário alargado nas USF, por diretiva da Administração Regional de Saúde (ARS), a partir do dia 1 de abril de 2012, fez com que os utentes com doença aguda não urgente passassem a poder recorrer, após as 20h, apenas às urgências hospitalares ou aos SASU. No entanto, não foram realizados estudos nacionais que avaliassem qual o melhor local para o atendimento de utentes com doença aguda não urgente, durante o alargamento de horário, o que revela ausência de fundamentação da medida tomada. Neste sentido, pretende-se quantificar e comparar a Perceção da Qualidade dos Serviços (PQS) e os custos (fármacos e meios complementares de diagnóstico - MCDT) entre os SASU e as USF.

A PQS é um constructo multidimensional, sendo que uma das formas de ser avaliada foi desenvolvida por Parasuraman e colaboradores, segundo o modelo «Expectativas-Perceções», por intermédio da utilização de um instrumento - SERVQUAL (22 items) -, tendo por base cinco componentes (elementos tangíveis, confiança no serviço, capacidade de resposta, confiança/garantia e empatia), a aplicar antes (para quantificar as expectativas) e após os serviços serem prestados (para quantificar as perceções). ${ }^{6-8}$ Posteriormente o instrumento foi simplificado por Cronin e colaboradores, que aplicam o mesmo instrumento, mas apenas após a prestação do serviço - SERVPERF -, demonstrando empírica e teoricamente a sua superioridade em relação à versão criada por Parasuraman, visão corroborada por outros autores. ${ }^{9-12}$ Vários autores referem a necessidade de uma adaptação do SERVQUAL/SERVPERF a cada contexto, nomeadamente ao da saúde, dadas as características quase únicas desta área. ${ }^{8,12-13}$ Foram construídos e validados instrumentos de aplicação hospitalar, tendo por base o SERVQUAL/SERVPERF, mas também de aplicação específica aos cuidados de saúde primários (CSP) - HEALTHQUAL. ${ }^{14-16}$

A prestação de cuidados de saúde deve ter em con- sideração não só a qualidade do serviço (técnica e funcional), mas também os custos a ela inerente..$^{13,17-18}$ Estudos realizados a nível nacional determinaram o custo/consulta nos CSP de $€ 39,68$ (fármacos e MCDT), sendo $€ 26,72(67,3 \%)$ referentes a custos com medicamentos e $€ 12,96(32,7 \%)$ a MCDT.*

O presente estudo apresenta como objetivos: 1) criar e testar a qualidade psicométrica de um instrumento de quantificação da PQS, adaptado aos CSP, em alargamento de horário, em contexto português; 2) quantificar e comparar a PQS dos utentes utilizadores de USF e SASU, em alargamento de horário; 3) determinar e comparar o custo de funcionamento (fármacos e MCDT) de USF e SASU em alargamento de horário. Deste modo, pretende-se analisar se o encerramento do alargamento de horário das USF terá sido a melhor medida, do ponto de vista dos custos e da PQS dos utentes.

\section{MÉTODOS}

\section{Desenho do estudo}

Estudo multicêntrico transversal descritivo e analítico em três etapas. Numa primeira parte foi construído e testado, do ponto de vista da sua qualidade psicométrica, um instrumento de medição da perceção da qualidade dos serviços (PQS). Este instrumento foi utilizado para quantificar a PQS dos utentes que recorrem à consulta aberta das USF e ao SASU. A segunda parte do estudo quantifica os custos de fármacos e MCDT, em ambas as unidades, durante o período em análise. Numa terceira fase, a PQS e custos com fármacos e MCDT são comparados entre USF e SASU.

Foram analisadas todas as unidades com alargamento de horário na zona de influência do Agrupamento de Centros de Saúde (ACeS) Porto Ocidental: USF Serpa Pinto (SP), USF Ramalde (RMD) e o SASU Porto (doravante designado apenas SASU), no sentido de quantificar os custos e avaliar a PQS dos utilizadores dos serviços prestados pelas unidades em alargamento de horário. O período de alargamento de horário das USF (em funcionamento até ao dia 01/04/2012, momento em que encerra) decorre da seguinte forma:

\footnotetext{
*Gouveia M, Silva SN, Oliveira P, Miguel LS. Análise dos custos dos centros de saúde e do regime remuneratório experimental. Grupo de Trabalho da Associação Portuguesa de Economia da Saúde; 2008. Documento de trabalho não publicado e disponibilizado aos autores.
} 
USF SP - $2^{\mathrm{a}}$ a $6^{\mathrm{a}}$ [20 às 23h] e aos sábados, domingos e feriados [15 às 20h]; USF RMD - $2^{\mathrm{a}}$ a $6^{\mathrm{a}}$ [20 às 00h] e aos sábados, domingos e feriados [08 às $00 \mathrm{~h}]$; SASU $-2^{\mathrm{a}}$ a $6^{\mathrm{a}}$ [20 às $00 \mathrm{~h}$ ] e aos sábados, domingos e feriados [09 às $21 \mathrm{~h}]$.

\section{Instrumento de estudo da PQS}

\section{$1^{a}$ Fase - Criação de instrumento de medição da PQS}

Para a avaliação da PQS foi criado um instrumento que teve por base o SERVPERF, HEALTHQUAL, os instrumentos criados por Carman e colaboradores, Choi e colaboradores, o parecer de peritos na área dos CSP e dos coordenadores de cada uma das unidades em estudo. ${ }^{12,14-16}$ Foi efetuado um pré-teste na USF SP, com cinco utentes, após o qual foi efetuada restruturação do questionário e nova avaliação pelos peritos. Foi feito novo pré-teste, na USF SP com quatro utentes, não havendo modificações a serem introduzidas. Posteriormente foram feitas adaptações ligeiras ao questionário para ir ao encontro das especificidades da USF RMD e do SASU, tendo sido aplicado um pré-teste com cinco utentes em cada uma das unidades, sem necessidade de alteração do instrumento. A versão definitiva do questionário encontra-se apresentada em anexo. Foram utilizados apenas quatro níveis de resposta («Discordo totalmente», «Discordo», «Concordo» $\mathrm{e}$ «Concordo totalmente») a cada um dos itens, ao contrário do que acontece com Carman e colaboradores (nove níveis de resposta), com SERVQUAL (sete níveis de resposta), SERVPERF (sete níveis de resposta) ou com HEALTHQUAL (cinco níveis de resposta). ${ }^{8,12,14,16} \mathrm{~A}$ reformulação da operacionalização das variáveis baseou-se no feedback do pré-teste, em que se verificou que as escalas numéricas de sete níveis (usadas originalmente no SERVQUAL/SERVPERF) eram de difícil compreensão para os utentes e mesmo as escalas nãonuméricas de cinco níveis (utilizadas no HEALTHQUAL, variando desde "Discordo totalmente» até «Concordo totalmente») não se revelaram totalmente adequadas, dada a incompreensão da hipótese de resposta «Não concordo nem discordo», pelo que se acabou por remover esta hipótese. Os itens, ao contrário do que foi feito no SERVQUAL e SERVPERF, foram, todos eles, colocados na forma afirmativa para facilitar a sua compreensão, tal como foi feito e recomendado no HEALTHQUAL. ${ }^{8,12,16}$

\section{$2^{a}$ Fase - Processo de simplificação por análise fatorial e tes- te da validade e fiabilidade do instrumento}

Foram obtidos um total de 221 questionários, sendo que destes foram excluídos todos os casos em que o respondente atribui a todos os itens do instrumento a mesma classificação, isto é, os participantes não discriminaram as diferentes dimensões do conceito $(n=83$, $37,6 \%$ ), pelo que passaram apenas a ser considerados 138 para análise de dados. Uma análise global da consistência interna (verifica se o instrumento determina resultados consistentes em sucessivas medições) determina um valor de de 0,9 (engloba todos os itens do instrumento), o que se entende como "ótimo". ${ }^{19}$ Foram avaliados os coeficientes de assimetria e achatamento, tendo sido obtidos valores dentro do intervalo de referência (assimetria inferior a $3 \mathrm{e}$ achatamento inferior a 8 , em valor absoluto ${ }^{20}$ para todos os itens, o que demonstra que a distribuição destes não se afasta de forma severa de uma distribuição normal. O valor de Kaiser-Meyer-Olkin (KMO) demonstra adequação da amostra para a análise pretendida: $\mathrm{KMO}=0,83$ (valor bom, dado que $0,8<\mathrm{KMO}<0,9$, demonstrando adequação da amostra para a realização de análise fatorial). ${ }^{21-22}$ O teste da esfericidade de Bartlett's $\chi^{2}(105)=1142,89$, $p<0,001$, indica que a correlação entre os itens é suficiente para realizar análise de componentes principais (ACP). ${ }^{22}$ Foi realizada uma ACP com os 15 itens do questionário, através de rotação ortogonal:Varimax (foi realizada igualmente ACP com rotação oblíqua: Oblimin, não havendo diferenças consideráveis nos pesos dos fatores extraídos, nem no seu agrupamento em componentes). Foram identificadas e consideradas para posterior análise quatro componentes, com eigen values superiores à unidade (critério de Kaiser), ${ }^{23}$ sendo que em conjunto explicavam $70,91 \%$ da variância (valor similar ao publicado na literatura).$^{16} \mathrm{O}$ Quadro I apresenta o peso de cada um dos fatores extraídos, após rotação. Os itens agrupados em cada um dos componentes sugerem que o componente 1 representa os «Profissionais de saúde», o componente 2 representa os «Rececionistas», o componente 3 representa o «Processo» (do inglês process) e o componente 4 «Tangíveis». A organização dos itens nos componentes apresentados vai ao encontro do já estabelecido na literatura. ${ }^{15-16} \mathrm{~A}$ consistência interna, dada pelo valor de de Cronbach, é de 0,9 para o componente «Profissionais de saúde» $\mathrm{e}$ «Rececionistas», 0,6 


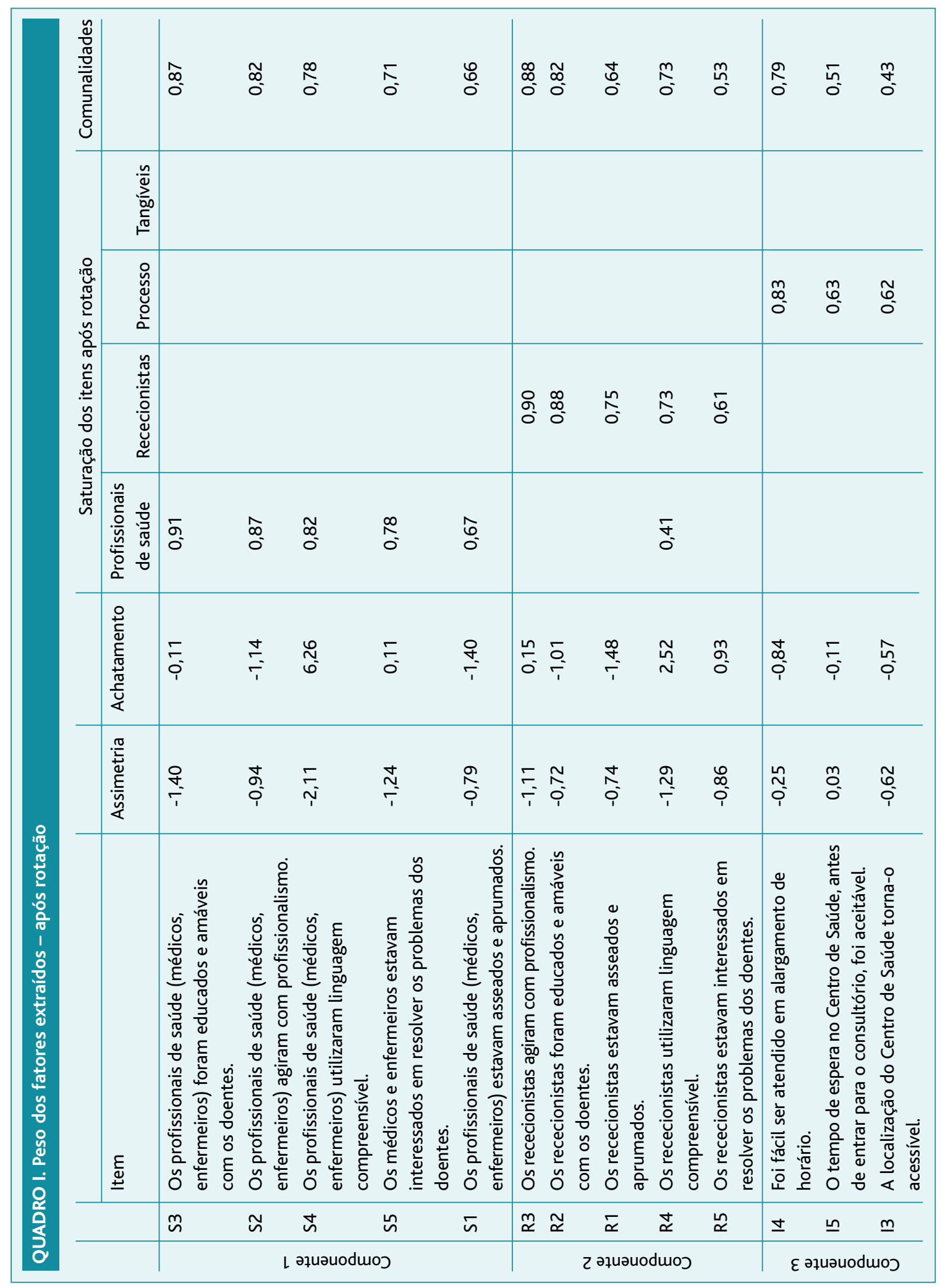




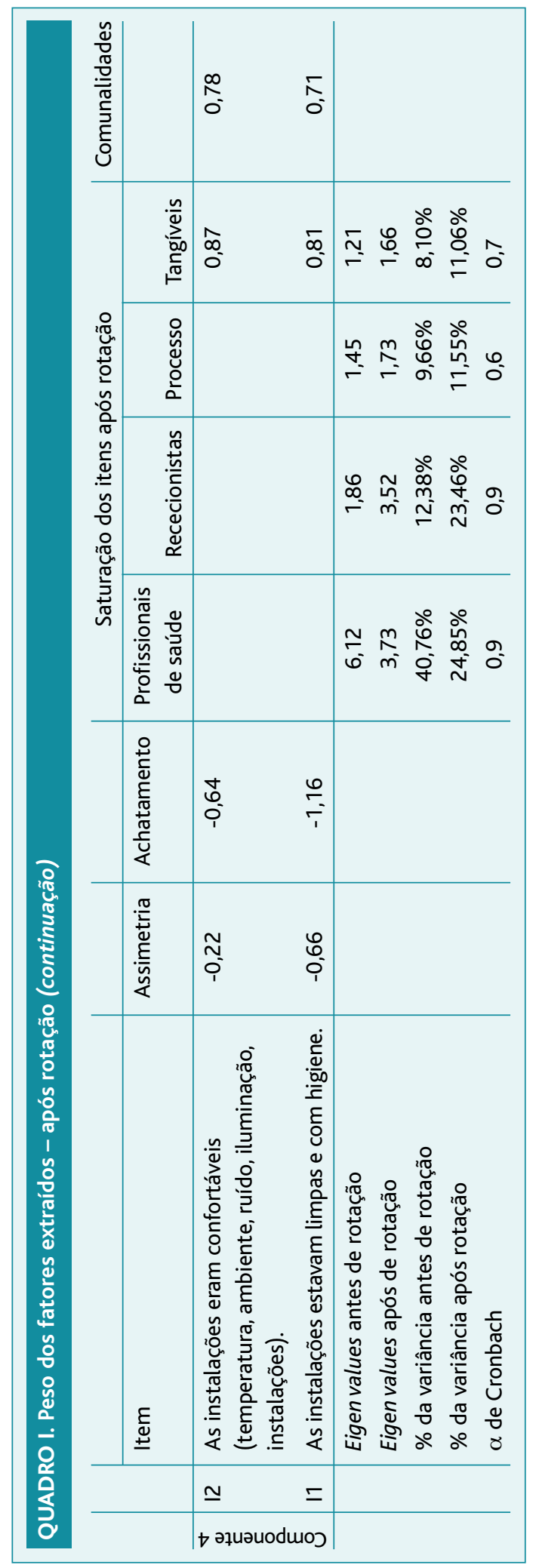

para o componente «Processo» e 0,7 para o componente «Tangíveis». Os valores obtidos são "ótimos" para o primeiro e segundo componentes, aceitável para o quarto componente, sendo menos satisfatório para o terceiro componente (ainda assim, aceitável, considerando o baixo número de itens), dado estarem a ser avaliados constructos psicológicos. ${ }^{19,24} \mathrm{~A}$ validade de conteúdo não foi avaliada, dado que a presente escala consiste numa adaptação do instrumento SERVQUAL/SERVPERF, que foi já extensivamente testado..$^{6-8,12}$

\section{Período de análise/Seleção dos utentes Análise de custos}

Para a determinação dos custos foram consideradas as prescrições (fármacos e MCDT) dos utentes que recorreram às USF e ao SASU durante o mês de outubro de 2011. O mês de outubro foi obtido de forma aleatória de entre um grupo de 15 meses, que engloba os 12 meses de 2011 (por uma questão de atualidade do trabalho científico, não foram incluídos anos anteriores), bem como os três primeiros meses de 2012 durante os quais ainda ocorreu o funcionamento, em alargamento de horário, das unidades de saúde.

\section{Perceção da qualidade dos serviços}

A medição da PQS nas USF foi realizada através de inquérito aos doentes que recorreram ao alargamento de horário nas USF de estudo, no ano prévio ao término do alargamento (31/03/2011 a 31/03/2012). O convite dos utentes para participação decorreu por conveniência, de forma presencial, nas USF de estudo, aos utentes com consulta agendada entre 03/07/2012 e 22/08/2012. Neste sentido, os utentes avaliam serviços de saúde que lhes foram prestados em momento anterior àquele em que são convidados a participar no estudo.

A determinação da PQS no SASU foi efetuada através de convites à participação dos utentes nos dias 6,8 e 10 de julho de 2012. Neste caso, os utentes avaliam a perceção de qualidade dos serviços que lhes foram prestados no dia em que são convidados a participar no presente estudo.

\section{Cálculo da dimensão amostral}

Análise de custos

$\mathrm{Na}$ análise de custos analisaram-se todos os utentes que recorreram às USF (275 utentes USF SP+132 uten- 
tes USF RMD) e uma amostra dos 5.347 utentes que recorreram ao SASU durante o período em análise. A amostra do SASU foi calculada tendo por base o custo médio/utente dos fármacos e MCDT das USF em comparação com os valores obtidos numa análise preliminar de 100 utentes no SASU. Por recurso ao GPower (v. 3.1, Gpower) foi determinado um effect size (d de Cohen) de 0,23 , o que determinou uma amostra necessária de 411 utentes do SASU (selecionados de forma aleatória de entre os 5.347 utentes).

\section{Perceção da qualidade dos serviços}

Foi considerada como necessária uma dimensão amostral mínima não inferior a 100, dadas as recomendações para a realização de análise fatorial exploratória. ${ }^{27}$

\section{Colheita e processamento de dados}

Para a determinação dos custos foram obtidos os números operacionais (NOP) dos utentes a estudar através do Sistema de Informação para as Unidades de Saúde (SINUS), o que permitiu aceder ao seu processo clínico por intermédio do Sistema de Apoio ao Médico (SAM). Dos processos clínicos foram extraídas as prescrições farmacológicas e MCDT, que foram posteriormente processadas em Excel (v. 2007, Microsoft Office Excel/v. 20, SPSS). As prescrições foram convertidas num custo (em Euros), mediante a utilização de preços de referência de cada um dos fármacos, publicados pelo INFARMED, bem como por consulta de tabela própria publicada pela ARS para o caso dos MCDT. ${ }^{25-26}$ Não foram contabilizados os custos dos fármacos de venda extra-formulário e não sujeitos a receita médica (MNSRM). No caso da mensuração da PQS, os NOP dos utentes de interesse foram obtidos através da plataforma de registo SINUS, o que permitiu ao secretariado entregar o questionário do estudo aos utentes previamente identificados e que se dirigiam, durante o período de realização do estudo, à sua USF por qualquer motivo. No caso do SASU, após a prestação das consultas, os utentes foram abordados pela equipa de investigação de forma a participarem no estudo. Os questionários foram aplicados durante todo o horário de funcionamento das unidades de saúde, durante os períodos temporais mencionados. Em todas as unidades foi assegurada a confidencialidade do processo, tendo sido criadas para o efeito caixas seladas, nas quais os questionários, anónimos (sem qualquer referência ao nome ou NOP do utente), eram depositados. Os resultados dos questionários foram processados em SPSS (v. 20, IBM SPSS Statistics).

Foram utilizados testes $t$ de Student e qui-quadrado para estudar as diferenças entre USF e SASU, do ponto de vista das características sociodemográficas, dos custos e da PQS. De forma a testar uma associação entre o modelo organizacional de prestação de cuidados (USF/SASU) e a PQS, de forma ajustada para possíveis variáveis de confundimento, foi determinado um modelo de regressão linear múltipla (Quadro IV). Foi considerado como variável dependente o valor de PQS e como independente o tipo de unidade. Esta análise foi ajustada para as variáveis idade, condição de trabalho e perceção de gravidade.

Foram considerados como pontos de referência para o effect size determinado os seguintes valores: 0,2 «Pequeno», 0,5 «Médio» e 0,8 «Grande», segundo o Cohen. ${ }^{28}$

\section{Revisão ética do projeto}

O protocolo de investigação foi submetido a aprovação da Coordenação do ACeS Porto Ocidental, Comissão de Ética da ARS Norte e à Comissão Nacional de Proteção de Dados. As duas primeiras instituições deram aval à realização do projeto, tendo a última considerado que não seria necessário a emissão de parecer.

\section{RESULTADOS}

\section{Determinação dos custos}

\section{Análise demográfica}

Durante o período em análise recorreram 275 pacientes à USF SP, 132 à USF RMD e 5.347 (dos quais apenas foram analisados 413, cerca de $8 \%$ ) ao SASU do Porto. A média das idades nas USF foi de 36 anos ( $\mathrm{DP}=25,2$, mínimo 0 e máximo 90) e de 33 anos no SASU ( $\mathrm{DP}=23,8$, mínimo 1 e máximo 89), estando os indivíduos distribuídos de forma sensivelmente uniforme pelos vários patamares etários, havendo predomínio em ambos os casos do patamar [0-19]. Verificou-se, em ambos os grupos, uma maior utilização pelos utentes do género feminino em relação ao masculino ( $66 \%$ vs $34 \%$, no caso das USF e $65 \%$ vs $31 \%$, no caso do SASU). Não foram encontradas diferenças estatisticamente significativas entre os grupos em análise (USF $v s$ SASU), seja na idade seja no género (Quadro II). 


\begin{tabular}{l}
\hline \multicolumn{2}{|l|}{ QUADRO II. Características sociodemográficas e custos - USF/SASU } \\
\hline \\
\hline Idade (anos)
\end{tabular}

vam algum critério de exclusão e cinco foram anulados pela equipa de investigação por inadequado preenchimento (os questionários com mais de três questões sem preenchimento foram anulados). Desta forma foi obtido um total de 221 questionários válidos, dos quais 104 prove-

\section{Análise de custos - fármacos e MCDT}

Na análise de custos globais (fármacos + MCDT) foi determinado um custo médio/utente de $€ 9,30(\mathrm{DP}=14,9)$ no caso das USF, sendo de $€ 8,20$ ( $D P=8,2)$ no SASU, não havendo no entanto diferenças estatisticamente significativas entre os grupos. Na análise dos custos com fármacos, o custo médio/utente foi de $€ 7,30$ (DP=12,1) no caso das USF e de $€ 8,00$ (DP=8,3) no SASU, não sendo também diferenças estatisticamente significativas entre ambos. No entanto, no caso dos custos associados aos MCDT verificou-se uma diferença estatisticamente significativa entre os grupos, com um custo médio de $€ 2,00$ (DP=9,1) no caso das USF e de $€ 0,20(\mathrm{DP}=1,2)$ no caso do SASU. Nas USF apenas $79 \%$ dos custos totais são com fármacos, no caso do SASU este valor ascende aos $97 \%$, havendo, portanto, neste último caso, um menor dispêndio em MCDT (Quadro II e Figura 1).

\section{Perceção da qualidade do serviço}

Foram aplicados um total de 281 questionários, dos quais 35 são referentes a indivíduos que se recusaram a colaborar no estudo, 20 pertencem a utentes que apresenta- nientes de SP, 18 de RMD e 99 do SASU. Após a eliminação dos questionários sem poder discriminante foram obtidos 68 em SP, 12 em RMD e 58 no SASU, perfazendo um total de 138.

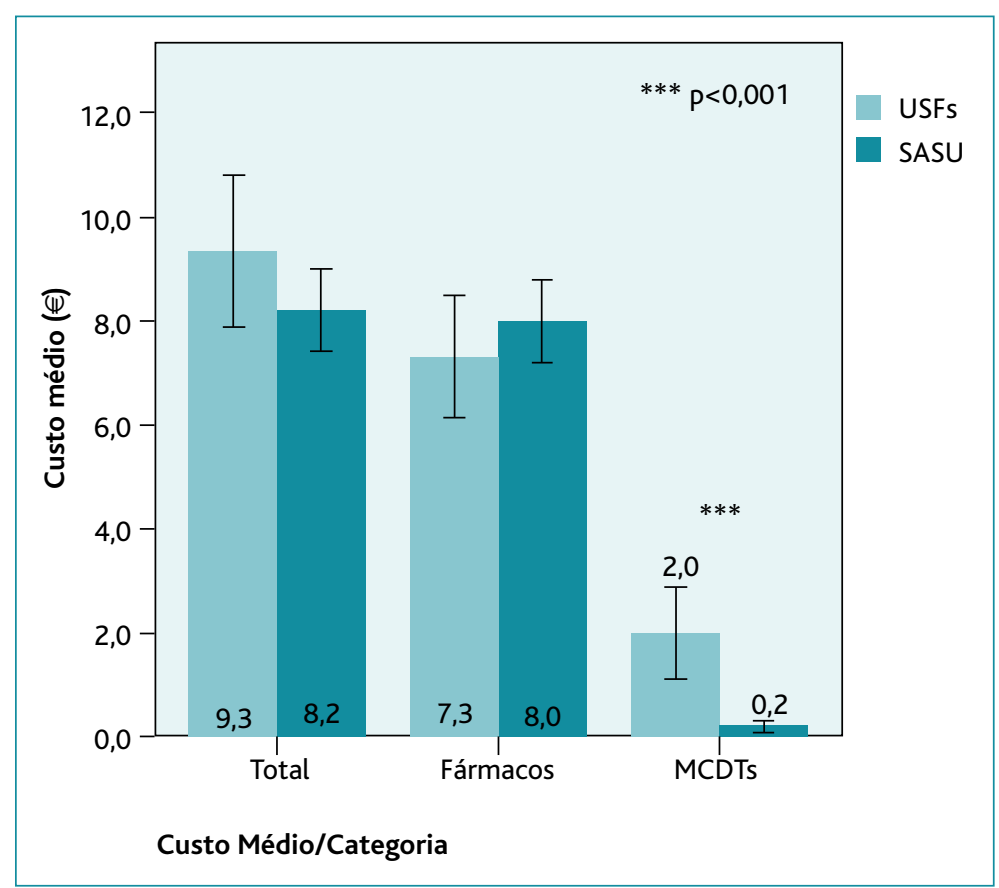

Figura 1. Comparação dos custos de atendimento de utentes, entre USF e SASU, em alargamento de horário, no Porto. 


\section{Análise demográfica}

No caso das USF, a média das idades dos respondentes é de 47 anos (DP=17,4), mínimo 18 e máximo 80, dos quais $68 \%$ são mulheres. Dos utentes, $26,8 \%$ tem idade entre os $18-39,36,6 \%$ entre os $40-59$ e $36,6 \%$ com 60 ou mais anos. No caso do SASU, a média das idades dos respondentes é de 40 anos (DP=15,9), mínimo 18 e máximo 88, dos quais $72 \%$ são mulheres. Dos utentes, $62,5 \%$ tem idade entre os $18-39,26,8 \%$ entre os $40-59$ e $10,7 \%$ com 60 ou mais anos. Nas USF, do ponto de vista da condição de trabalho, 54,9\% dos respondentes está ativo (empregado/estudante), $16,9 \%$ desempregado, $28,2 \%$ reformado. No caso dos SASU, $61,1 \%$ está ativo, 27,8\% desempregado e $11,1 \%$ re- formado. Na avaliação do nível de estudos, no caso das USF, $1,4 \%$ tinha $\leq 4$ anos de estudos, $34,3 \%$ quatro anos de estudos, 22,9\% nove anos de estudos, $24,3 \%$ doze anos de estudos e $17,1 \%$ formação superior. No caso dos SASU, nenhum utente tinha $\leq$ quatro anos de estudos, $17,5 \%$ quatro anos de estudos, $26,3 \%$ nove anos de estudos, $36,9 \%$ doze anos de estudos e 19,3\% formação superior. Nas USF, $59 \%$ dos utentes considerou a sua doença como sendo «Grave» e $41 \%$ «Não grave», enquanto no caso do SASU $33,9 \%$ considerou a sua doença «Grave» e $66,1 \%$ «Não grave». Apenas foram encontradas diferenças estatisticamente significativas para a idade, condição de trabalho e perceção de gravidade (Quadro III).

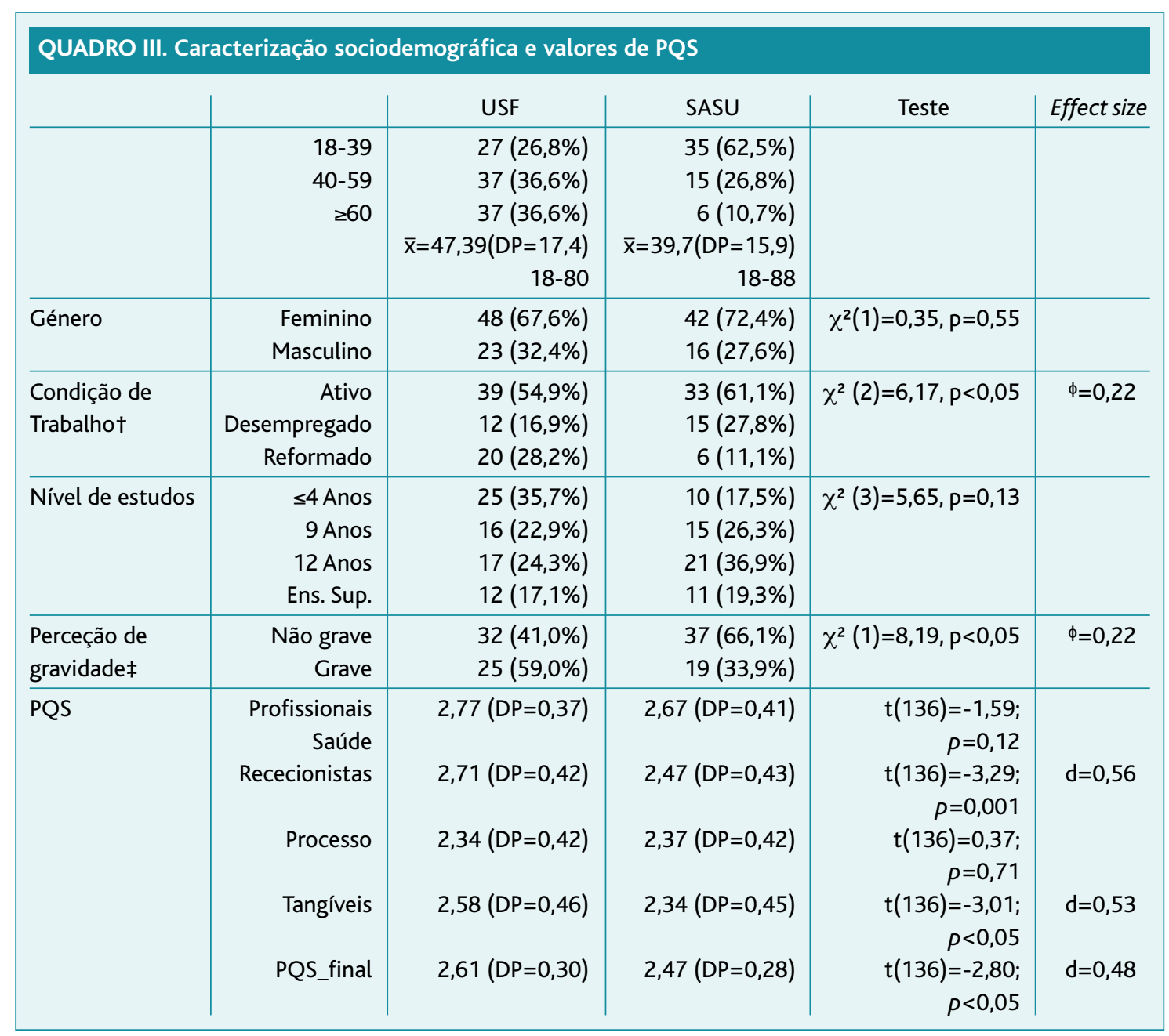

† A variável foi agrupada. O grupo ativo engloba os utentes «Estudantes» ou «Empregados».

¥ A variável foi agrupada. O grupo não grave engloba os utentes que reportaram «Nada grave» ou «Pouco grave» e o grupo grave engloba «Grave» e «Extremamente grave». 


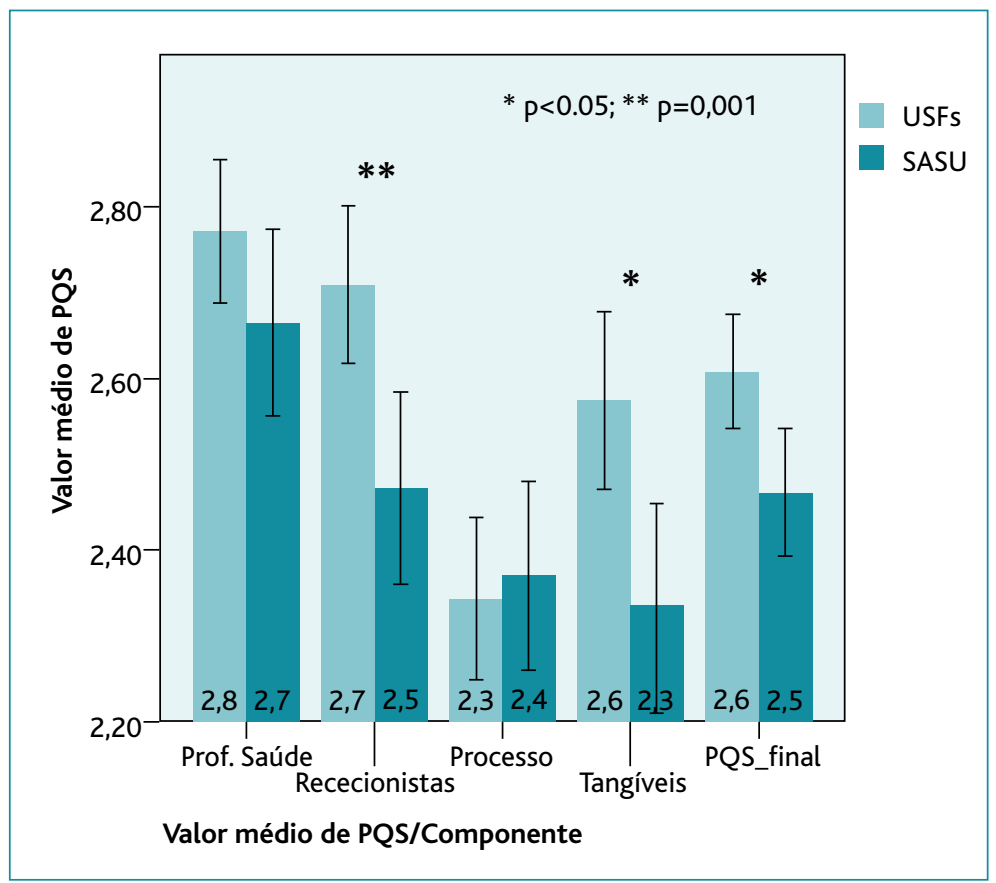

Figura 2. Comparação dos valores de satisfação e dos seus componentes, entre USF e SASU, em alargamento de horário, no Porto.

\section{Comparação USF vs SASU}

Foram comparados os scores obtidos pelas USF com os obtidos pelo SASU, para cada um dos itens, do instrumento criado. Há uma superioridade das USF em relação ao SASU, nos seus valores médios, em 13 dos 15 itens do instrumento: «Limpeza» (I1), «Conforto» (I2), «Acessibilidade» (I3), «Facilidade de atendimento» (I4), todos os itens de avaliação dos rececionistas (R1-R5), e o "Asseio» (S1), «Profissionalismo» (S2), «Educação» (S3) e «Uso de linguagem compreensível» (S4) pelos profissionais de saúde. Há uma superioridade do SASU nos itens «Tempo de espera antes de entrar no consultório» (I5) e «Interesse dos profissionais de saúde em resolver os problemas dos utentes» (S5). Verificou-se a existência de uma diferença estatisticamente significativa para os itens «Conforto» (I2), «Tempo de espera antes de entrar no consultório» (I5), «Educação dos rececionistas» (R2), «Uso de linguagem compreensível pelos rececionistas» (R4), «Interesse dos rececionistas em resolver os problemas» (R5) e "Asseio dos profissionais de saúde» (S1), associados a um effect size médio. Na análise dos componentes extraídos verificou-se igualmente uma superioridade das USF sobre o SASU no componente «Profissionais de saúde», «Rececionistas» $\mathrm{e}$ «Tangíveis», sendo as diferenças estatisticamente significativas para os últimos dois componentes mencionados (effect size médio). A superioridade do SASU sobre as USF verificada no componente «Processo» não é estatisticamente significativa. Do ponto de vista do scoreglobal calculado (PQS_final), que engloba os quatro componentes extraídos, verifica-se uma superioridade estatisticamente significativa das USF sobre o SASU (USF 2,61 e SASU 2,47, $p<0,05$, quase atingindo um valor de effect size médio) (Quadro III e Figura 2).

\section{Comparação da PQS entre grupos de forma ajustada}

O modelo de regressão linear múltiplo revelou um valor da ANOVA significativo $[\mathrm{F}(5,114)=2,51, p<0,05]$, demonstrando ser melhor preditor do que o valor médio. No modelo apresentado, apenas o tipo de unidade tem valor preditor significativo da variável dependente. O modelo evidencia que, mantendo constante todas as variáveis para a variável Unidade de Saúde, as USF são responsáveis por uma maior contribuição no valor de PQS do que o SASU (mais 0,16 unidades, numa escala de 0 a 3 ).

\section{DISCUSSÃO}

Perceção da qualidade dos serviços

Utilizando o questionário construído e testado do ponto de vista da sua qualidade psicométrica, observou-se uma superioridade estatisticamente significativa nos valores de PQS das USF em relação ao SASU. Numa análise mais detalhada verificou-se uma superioridade estatisticamente significativa das USF sobre o SASU nos itens I2, R2, R4, R5, S1, nos componentes «Rececionistas» $\mathrm{e}$ «Tangíveis» e no score global, o que evidencia uma superioridade das USF, do ponto de vista das perceções da qualidade dos serviços. É de sublinhar o facto de os três itens cotados com melhor score serem correspondentes aos profissionais de saúde da USF: «Profissionalismo» (S2), «Educação» (S3) e «Uso de linguagem compreensível» (S4). No entanto, os itens com pior score foram: «Tempo de espera antes de en- 
QUADRO IV. Regressão - PQS

Equação do modelo de PQS Global: $\breve{Y}=2,261+0,160 \chi_{1}+0,001 \chi_{2}+0,072 \chi_{3}-0,104 \chi_{4}+0,041 \chi_{5}$

$R_{\text {(não ajustado) }}=10,0 \% ; R_{\text {(ajustado) }}^{2}=5,9 \%$

$F(5,114)=2,51, p<0,05$

\begin{tabular}{l|c|c|c|c|c|c|c}
\hline & $\begin{array}{c}\text { B (não } \\
\text { estandardizado) }\end{array}$ & $\begin{array}{c}\text { Erro estandardizado } \\
\text { de B }\end{array}$ & $\begin{array}{c}\text { B } \\
\text { (estandardizado) }\end{array}$ & $t$ & Sig. de $t$ & \multicolumn{2}{|l}{$\begin{array}{c}\text { Intervalo de confiança 95\% } \\
\text { de B (não estandardizado) }\end{array}$} \\
\hline Constante & 2,261 & 0,120 & & & & \\
\hline Unidade Saúde $\left(\chi_{1}\right)$ & 0,160 & 0,054 & 0,28 & 2,981 & $p<0,05$ & 0,052 & 0,257 \\
\hline Idade $\left(\chi_{2}\right)$ & 0,001 & 0,002 & 0,04 & 0,188 & $p=0,810$ & $-0,003$ & 0,004 \\
\hline Desempregado $\left(\chi_{3}\right)$ & 0,072 & 0,064 & 0,103 & 1,701 & $p=0,266$ & $-0,014$ & 0,191 \\
\hline Reformado $\left(\chi_{4}\right)$ & $-0,104$ & 0,106 & $-0,145$ & $-0,851$ & $p=0,329$ & $-0,150$ & 0,060 \\
\hline Gravidade $\left(\chi_{5}\right)$ & 0,041 & 0,054 & 0,071 & 0,790 & $p=0,449$ & $-0,051$ & 0,130 \\
\hline
\end{tabular}

$\chi_{1}$ - SASU (referência) e USF (teste)

$\chi_{3}-$ Não desempregado (referência) e desempregado (teste)

$\chi_{4}-$ Não reformado (referência) e reformado (teste)

$\chi_{5}$ - foram criados dois grupos: grupo 0 (respostas «Nada grave» e «Pouco grave»), grupo 1 (respostas «Grave» e «Extremamente grave»)

trar no consultório» (I5) da USF e do SASU e «Conforto das instalações» (I2) do SASU.

A criação do modelo de determinação da PQS reforçou a superioridade das USF sobre o SASU, dado que mesmo quando são controladas as variáveis estatisticamente diferentes entre os grupos (idade, condição de trabalho e perceção de gravidade), as USF continuam a reportar maiores valores de PQS (mais 5,3 unidades numa escala de 0 a 100). Não foi possível identificar a idade, condição de trabalho ou perceção de gravidade como preditores da PQS, por oposição ao descrito na literatura sobre os preditores sociodemográficos da PQS/satisfação para os cuidados de saúde..$^{29-33}$ No entanto, não poderá ser excluído o confundimento introduzido por variáveis como o grau de diferenciação e especialização dos profissionais das unidades, o tempo de serviço ou o tipo de contrato, a relação doentemédico-unidade, para as quais a análise não foi controlada.

Foi observado um claro desvio-direito dos resultados, em ambas as unidades, em todos os itens do instrumento (concentração das respostas nos dois níveis superiores «Concordo» $\mathrm{e}$ «Concordo totalmente», compreendendo $98,6 \%$ das respostas nestes dois patama- res), demonstrando altas perceções de qualidade, o que vai ao encontro de outros estudos. ${ }^{16}$

A análise da PQS foi realizada imediatamente após a prestação dos serviços de saúde, no caso do SASU. No caso das USF, a avaliação da perceção da PQS reporta-se ao momento de prestação prévio, havendo assim uma comparação de utentes que não recorreram no mesmo período temporal às unidades de saúde, não se podendo descartar viés de memória no caso das USF. Acresce ao mencionado ter sido utilizada uma amostra de conveniência em ambas as unidades. Estas limitações podem ser superadas em estudos posteriores, mediante a utilização de uma amostragem aleatória, inquirindo telefonicamente os utentes, eliminando também a questão do viés de memória, que existiu no caso da USF.

\section{Quantificação dos custos}

Foi determinado um custo médio de atendimento (fármacos+MCDT) de $€ 9,30$ ( $\mathrm{DP}=14,9$ ), no caso das USF e de $€ 8,20$ ( $D P=8,2$ ), no caso do SASU, não havendo, no entanto, diferenças estatisticamente significativas entre os grupos. É notória maior prescrição de MCDT no caso das USF do que no SASU ( $22 \%$ vs $3 \%$ dos 
custos totais, respetivamente). Após análise dos processos clínicos verificou-se que, enquanto no SASU os MCDT se encontram direcionados para o estudo de doença aguda (e.g., R-X), no caso das USF há também a prescrição de MCDT para o estudo de doença não aguda (PSA/perfil lipídico). Este procedimento torna-se justificável pelo facto de o utente estar fidelizado às USF e não aos SASU, o que faz com que possam ser solicitados pelos clínicos MCDT enquadrados em planos de vigilância anual. Neste sentido, numa análise apenas dos custos associados a fármacos verifica-se que $o$ custo médio de atendimento é de $€ 7,30(\mathrm{DP}=12,1)$ nas USF e de $€ 8,00$ (DP=8,3) no SASU, o que evidencia um atendimento mais barato nas USF, não sendo, no entanto, a diferença estatisticamente significativa. Para ambas as unidades os custos médios calculados são inferiores aos determinados a nível nacional para os CSP. Os custos apresentados apenas consideram fármacos e MCDT; no entanto, na ótica do sistema de saúde seria também relevante considerar, em posterior trabalho, outros custos diretos ou indiretos, como custo/hora dos profissionais de saúde, fornecimento de serviços externos (limpeza e segurança) ou rendas para uma análise mais exaustiva desta comparação.

\section{Limitações}

Os resultados de PQS e custos com o atendimento dos utentes encontram-se influenciados por fatores não considerados na presente análise. No caso das USF, a medição da exposição poderá estar a ser alvo de viés de memória, dado que os utentes avaliam serviços que lhes foram prestados em momento prévio ao que são inquiridos, o que não ocorre no caso do SASU. Adicionalmente, dado que os utentes que recorrem ao SASU avaliam os serviços que lhes são prestados em julho e os que recorrem às USF avaliam serviços prestados em qualquer mês de 2012, tal pode condicionar a satisfação, dado que o mês condiciona a afluência aos serviços, bem como a disponibilidade de escalas de profissionais e, consequentemente, a PQS. Este fenómeno de sazonalidade poderá também condicionar os custos que foram determinados.

\section{CONCLUSÃO}

Os valores globais de PQS nas USF são superiores aos determinados para os SASU, sendo esta diferença estatisticamente significativa, mesmo quando é controlado o peso das características sociodemográficas. Não foram encontradas diferenças estatisticamente significativas entre os custos globais (fármacos+MCDT) das USF e do SASU. Não há, assim, evidência a favor do encerramento do alargamento de horário nas USF, dado apresentarem superioridade do ponto de vista da PQS e custos similares aos apresentados pelo SASU.

\section{AGRADECIMENTOS}

Os autores gostariam de agradecer à colaboração institucional de: Dr. Rui Medon, Diretor Executivo do ACeS Porto Ocidental Dra. Maria Emília Penêda, Coordenadora do SASU Porto Dra. Ana Castanheira, Coordenadora da USF Serpa Pinto Dr. Dilermando Sobral, Coordenador da USF Ramalde

\section{REFERÊNCIAS BIBLIOGRÁFICAS}

1. van Uden CJ,Ament AJ, Voss GB, Wesseling $G$, Winkens RA, van Schayck OC, et al. Out-of-hours primary care: implications of organisation on costs. BMC Fam Pract. 2006;7:29.

2. Leibowitz R, Day S, Dunt D. A systematic review of the effect of different models of after-hours primary medical care services on clinical outcome, medical workload, and patient and GP satisfaction. Fam Practice. 2003;20(3):311-7.

3. Penson R, Coleman P, Mason S, Nicholl J. Why do patients with minor or moderate conditions that could be managed in other settings attend the emergency department? Emerg Med J. 2012;29(6):487-91.

4. Gentile S, Vignally P, Durand AC, Gainotti S, Sambuc R, Gerbeaux P. Nonurgent patients in the emergency department? A French formula to prevent misuse. BMC Health Serv Res. 2010;10:66.

5. Lega F, Mengoni A. Why non-urgent patients choose emergency over primary care services? Empirical evidence and managerial implications. Health Policy. 2008;88(2-3):326-38.

6. Zeithaml VA, Berry LL, Parasuraman A. Communication and control processes in the delivery of service quality. J Mark. 1988;52(2):35-48.

7. Parasuraman A, ZeithamIVA, Berry LL. A conceptual model of service quality and its implications for future research. J Mark. 1985;49(4):41-50.

8. Parasuraman A, ZeithamIVA, Berry LL. SERVQUAL: a multiple-item scale for measuring consumer perceptions of service quality. J Retailing. 1988;64(1):12-40.

9. Jain SK, Gupta G. Measuring service quality: SERVQUAL vs. SERVPERF scales. VIKALPA. 2004;29(2):25-37.

10. Babakus E, Boller GW. An empirical assessment of the SERVQUAL scale. J Business Res. 1992;24(3):253-68.

11. Parasuraman A, Zeithaml VA, Berry LL. Reassessment of expectations as a comparison standard in measuring service quality: Implications for further research. J Mark. 1994;58(1):111-24.

12. Cronin JJ Jr, Taylor SA. Measuring service quality: a reexamination and extension. J Mark. 1992;56(3):55-68.

13. Donabedian A. The quality of care: how can it be assessed? JAMA. 1988;260(12):1743-8.

14. Carman JM. Patient perceptions of service quality: combining the dimensions. J Serv Mark. 2000;14(4):337-52 
15. Choi K, Cho W, Lee S, Lee H, Kim C. The relationships among quality, value, satisfaction and behavioral intention in health care provider choice. J Bus Res. 2004;57(8):913-21.

16. Miranda FJ, Chamorro A, Murillo LR, Vega J. Assessing primary healthcare services quality in Spain: managers vs patients perceptions. Serv Ind J. 2010;30(13):2137-49.

17. Kringos DS, Boerma WG, Hutchinson A, Van der Zee J, Groenewegen PP. The breadth of primary care: a systematic literature review of its core dimensions. BMC Health Serv Res. 2010;10:65.

18. Grönroos C.A service quality model and its marketing implications. Eur J Mark. 1984;18(4):36-44.

19. Kline P. The handbook of psychological testing. 2nd ed. London: Routledge; 1999. ISBN 9780415211581

20. Klein RB. Data preparation. In: Kline RB, editor. Principles and practice of structural equation modeling. 3rd ed. New York: Guilford Press; 2010. p. 46-74. ISBN 9781606238769

21. Hutcheson G, Sofroniou N. Factor analisys. In: Hutcheson G, Sodroniou $\mathrm{N}$, editors. The multivariate social scientist: introductory statistics using generalized linear models. London: Sage; 2009. p. 217-52. ISBN 9780761952015

22. Malhotra N. Factor analysis. In: Malhotra N, editor. Marketing research: an applied orientation. 3rd ed. New Jersey: Pearson Education; 1999. p. 585-609. ISBN 9780130830449

23. Kaiser HF. The application of electronic computers to factor analysis. Educ Psychol Meas. 1960;20(1):141-51.

24. Hair JF, Anderson RE, Tatham RL, Black WC. Multivariate data analysis with readings. 4th ed. New Jersey: Prentice-Hall; 1995. ISBN 9780139133107

25. Administração Central do Sistema de Saúde. MCDTs convencionados [Internet]. Lisboa: ACSS; 2012 [cited 2012 Feb]. Available from: http://www.acss.min-saude.pt/DownloadsePublicações/Tabelaselmpressos/Convencionados/tabid/143/language/pt-PT/Default.aspx

26. INFARMED. Pesquisa do preço de medicamentos [Internet]. Lisboa: INFARMED; 2012 [cited 2012 Feb]. Available from: http://www.infarmed.pt/genericos/pesquisamg/pesquisaMG.php

27. Kline P. Psychometrics and psychology. London:Academic Press; 1979. ISBN 9780124151505

28. Cohen J.The $t$ test for means. In: Cohen J, editor. Statistical power analysis for the behavioral sciences. 2nd ed. New Jersey: Routledge; 1988. p. 19-74. ISBN 9780805802832
29. Hall JA, Dornan MC. Patient sociodemographic characteristics as predictors of satisfaction with medical care: a meta-analysis. Soc Sci Med. 1990;30(7):811-8.

30. Cohen G. Age and health status in a patient satisfaction survey. Soc Sci Med. 1996;42(7):1085-93.

31. Sun BC, Adams JG, Burstin HR. Validating a model of patient satisfaction with emergency care. Ann Emerg Med. 2001;38(5):527-32.

32. Rahmqvist M. Patient satisfaction in relation to age, health status and other background factors: a model for comparisons of care units. Int J Qual Health Care. 2001;13(5):385-90.

33. Butler D, Oswald SL, Turner DE. The effects of demographics on determinants of perceived health-care service quality: The case of users and observers. J Manag Med. 1996;10(5):8-20.

34. Cars $\mathrm{O}$, Mölstad S, Melander A. Variation in antibiotic use in the European Union. Lancet. 2001;357(9271):1851-3.

35. Fernández-Liz E, Modamio P, Catalán A, Lastra CF, Rodríguez T, Mariño EL. Identifying how age and gender influence prescription drug use in a primary health care environment in Catalonia, Spain. Br J Clin Pharmacol. 2008;65(3):407-17.

36. Maroco J. Análise de regressão linear. In: Maroco J, editor. Análise estatística com utilização do SPSS. Lisboa: Sílabo; 2003. p. 373-465. ISBN 9789726184522

37. Field A. Regression. In: Field A, editor. Discovering statistics using SPSS. 3rd ed. London: Sage; 2009. p. 197-263. ISBN 9781847879073

\section{CONFLITOS DE INTERESSES}

Os autores não têm nenhum conflito de interesses a declarar.

\section{FINANCIAMENTO DO ESTUDO}

Este estudo não foi apoiado por nenhum subsídio ou bolsa.

\section{COMISSÃO DE ÉTICA}

Estudo realizado após parecer favorável da Comissão de Ética da ARS Norte.

\section{ENDEREÇO PARA CORRESPONDÊNCIA}

João Firmino-Machado

E-mail: firmino.firminomachado@gmail.com

Recebido em 28-04-2016

Aceite para publicação em 13-02-2017 


\section{ABSTRACT \\ THE IDEAL SITE FOR PROVISION OF OUT-OF-HOURS CARE IN PRIMARY CARE: AN ANALYSIS OF COSTS AND PERCEPTION OF QUALITY OF SERVICES}

Objective: This study aims to compare the costs of out-of-hours services and patient Perceptions of Service Quality (PSQ), between a regular primary care units and a specialized unit only opened to perform this kind of care.

Type of study: Cross-sectional - analytical and observational.

Local: Unidade de Saúde Familiar (USF) Serpa Pinto, USF Ramalde and Serviço de Atendimento de Situações Urgentes (SASU) do Porto, from Agrupamento de Centros de Saúde Porto Ocidental.

Population: Patients who attend out-of-ours services at the enrolled health units.

Methods: A questionnaire developed by the research team was used to determine PSQ. The SERVQUAL/SERVPERF instruments and their adaptations to health sector, particularly HEALTHQUAL, were used as models. The instrument was applied to 281 patients in both units. The comparison of costs between units was determined through the analysis of 820 clinical records. National reference values were used to calculate costs of drugs and laboratory tests.

Results: Significantly higher values of perception of service quality were found among patients treated in the USF compared to SASU, controlling for the patient's socio-demographic characteristics. No significant differences were found in costs between units. The mean costs per patient in the USF were $€ 9.3$ ( $S D=14.9)$ compared to $€ 8.2(S D=8.2)$ for the SASU.

Discussion/Conclusion: If high quality after hours care with greater patient satisfaction can be provided in family health units at similar costs to care in emergency units, there is no evidence in favour of closing after hours care services in family health units.

Keywords: Patient satisfaction; Primary health care; Health care costs; After-hours care. 